\title{
Existence and monotone iteration of unique solution for tempered fractional differential equations Riemann-Stieltjes integral boundary value problems
}

\author{
Bibo Zhou ${ }^{1,2}$, Lingling Zhang ${ }^{1,3 *}$, Nan Zhang ${ }^{1}$ and Emmanuel Addai ${ }^{1}$
}

"Correspondence: tyutzl|@126.com ${ }^{1}$ College of Mathematics, Taiyuan University of Technology, Taiyuan P.R. China

${ }^{3}$ State Key Laboratory of Explosion Science and Technology, Beijing Institute of Technology, Beijing, P.R. China

Full list of author information is available at the end of the article

\begin{abstract}
The primary objective of this research paper is to investigate two kinds of high-order tempered fractional differential equations integral boundary value problems. By means of the mixed monotone operators fixed point theorems with perturbation and the increasing $\varphi-(h, \sigma)$-concave operators fixed point theorems, we can not only guarantee the existence-uniqueness of solution, but also construct successively sequences for approximating the unique solution. In addition, we demonstrate the effectiveness of the main result by using two examples.
\end{abstract}

Keywords: Existence-uniqueness; Tempered fractional differential equations; Mixed monotone operator; $\varphi-(h, \sigma)$-concave operators; Riemann-Stieltjes integral boundary value conditions

\section{Introduction}

In this paper, we investigate the following tempered fractional differential equations Riemann-Stieltjes integral boundary values problems (BVP for short):

$$
\left\{\begin{array}{l}
{ }_{0}^{R} \mathbb{D}_{t}^{\alpha, \lambda} u(t)+f(t, u(t), u(t))+g(t, u(t))=0, \quad t \in(0,1), \\
u(0)={ }_{0}^{R} \mathbb{D}_{t}^{\gamma_{1}, \lambda} u(0)={ }_{0}^{R} \mathbb{D}_{t}^{\gamma_{2}, \lambda} u(0)=\cdots={ }_{0}^{R} \mathbb{D}_{t}^{\gamma_{n-2}, \lambda} u(0)=0, \\
{ }_{0}^{R} \mathbb{D}_{t}^{\beta_{1}, \lambda} u(1)=\int_{0}^{\eta} b(s){ }_{0}^{R} \mathbb{D}_{t}^{\beta_{2}, \lambda} u(s) d A(s)+\int_{0}^{1} a(s){ }_{0}^{\beta_{3}, \lambda} u(s) d A(s)
\end{array}\right.
$$

and

$$
\left\{\begin{array}{l}
{ }_{0}^{R} \mathbb{D}_{t}^{\alpha, \lambda} u(t)+\psi(t, u(t))=c, \quad t \in(0,1), \\
u(0)={ }_{0}^{R} \mathbb{D}_{t}^{\gamma_{1}, \lambda} u(0)={ }_{0}^{R} \mathbb{D}_{t}^{\gamma_{2}, \lambda} u(0)=\cdots={ }_{0}^{R} \mathbb{D}_{t}^{\gamma_{n-2}, \lambda} u(0)=0, \\
{ }_{0}^{R} \mathbb{D}_{t}^{\beta_{1}, \lambda} u(1)=\int_{0}^{\eta} b(s){ }_{0}^{R} \mathbb{D}_{t}^{\beta_{2}, \lambda} u(s) d A(s)+\int_{0}^{1} a(s){ }_{0}^{R} \mathbb{D}_{t}^{\beta_{3}, \lambda} u(s) d A(s),
\end{array}\right.
$$

(c) The Author(s) 2020. This article is licensed under a Creative Commons Attribution 4.0 International License, which permits use, sharing, adaptation, distribution and reproduction in any medium or format, as long as you give appropriate credit to the original author(s) and the source, provide a link to the Creative Commons licence, and indicate if changes were made. The images or other third party material in this article are included in the article's Creative Commons licence, unless indicated otherwise in a credit line to the material. If material is not included in the article's Creative Commons licence and your intended use is not permitted by statutory regulation or exceeds the permitted use, you will need to obtain permission directly from the copyright holder. To view a copy of this licence, visit http://creativecommons.org/licenses/by/4.0/. 
where ${ }_{0}^{R} \mathbb{D}_{t}^{\alpha, \lambda} u,{ }_{0}^{R} \mathbb{D}_{t}^{\gamma_{k}, \lambda} u(k=1,2, \ldots, n-2)$ and ${ }_{0}^{R} \mathbb{D}_{t}^{\beta_{i}, \lambda} u(i=1,2,3)$ are the tempered fractional derivatives defined by

$$
{ }_{0}^{R} \mathbb{D}_{t}^{\alpha, \lambda} u(t)=e_{0}^{-\lambda t R} D_{t}^{\alpha}\left(e^{\lambda t} u(t)\right), \quad \lambda \geq 0 .
$$

Here, ${ }_{0}^{R} D_{t}^{\alpha}$ denotes the standard Riemann-Liouville fractional derivative

$$
{ }_{0}^{R} D_{t}^{\alpha} u(t)=\frac{d^{n}}{d t^{n}}\left({ }_{0} I_{t}^{n-\alpha} u(t)\right)
$$

where ${ }_{0} I_{t}^{v}$ for $v>0$ is the fractional integral operator of order $v$ defined by

$$
{ }_{0} I_{t}^{v} \psi=\frac{1}{\Gamma(v)} \int_{0}^{t}(t-s)^{v-1} \psi(s) d s
$$

In addition, $n-1<\alpha \leq n, c>0$ is a constant, $k-1<\gamma_{k} \leq k(k=1,2, \ldots, n-2), n-k-1<$ $\alpha-\gamma_{k} \leq n-k(k=1,2, \ldots, n-2), \beta_{1} \geq \beta_{2}, \beta_{1} \geq \beta_{3}, \alpha-\beta_{i}>1(i=1,2,3), a, b \in C(0,1)$, $A$ is a function of bounded variation, $\int_{0}^{\eta} b(s)_{0}^{R} \mathbb{D}_{t}^{\beta_{2}} u(s) d A(s)$ and $\int_{0}^{1} a(s)_{0}^{R} \mathbb{D}_{t}^{\beta_{3}} u(s) d A(s)$ denote Riemann-Stieltjes integral with respect to $A$. By using some fixed point theorems, we establish sufficient conditions that ensure the existence-uniqueness of solutions for BVP (1.1) and BVP (1.2), respectively.

Over the past few decades, more theories and experiments show that a great deal of abnormal phenomena that occur in the applied science and engineering can be described by fractional differential equations; therefore, fractional differential equations arise in lots of fields such as economics, mechanics, physics, chemistry, biological science, etc.; see [14] for example. It is because the fractional order derivatives provide power tools for the description of memory and hereditary characteristics of different processes and materials in many fields.

In addition, in order to increase the power and applicability of the fractional calculus, Caputo and Fabrizio have recently defined a new fractional derivative possessing a singular kernel [5]. Some researchers have used distinct methods for solving some different equations including the Caputo-Fabrizio fractional derivative (see [6-11]). For instance, Aydogan and Baleanu [6] investigated the existence of solutions for two highorder fractional differential equations including the Caputo-Fabrizio derivative. In [7], Baleanu et al. extended the fractional Caputo-Fabrizio derivative of order $0 \leq \sigma<1$ on $C_{\mathbb{R}}[0,1]$ and investigated two high-order series-type fractional differential equations involving the extended derivation. In [8], a new fractional model for human liver involving Caputo-Fabrizio derivative with exponential kernel was introduced. By using the fractional Caputo-Fabrizio derivative, Aydogan et al. [12] introduced two types of new highorder derivations called CFD and DCF and investigated the existence of solutions for such two types of high-order fractional integro-differential equations. In [13], the authors showed that four fractional integro-differential inclusions had solutions. Also, it has been proved that working with the Caputo-Fabrizio fractional derivative is much better than with other fractional derivatives (the reader can see, for example, [14-17] and the references therein). 
In [18], the author considered a class of boundary value problems of Caputo tempered fractional differential equations as follows:

$$
\left\{\begin{array}{l}
{ }_{0}^{C} \mathbb{D}_{t}^{\theta, \lambda} u(z)=g(z, u(z)), \quad z \in[0, T], \\
a u(0)+b e^{\lambda T} u(T)=c,
\end{array}\right.
$$

where $g(z, u(z)):[0, T] \times \mathbb{R} \rightarrow \mathbb{R}$ is a continuous function, ${ }_{0}^{C} \mathbb{D}_{t}^{\theta, \lambda}$ is the Caputo tempered fractional derivative defined by ${ }_{0}^{C} \mathbb{D}_{t}^{\theta, \lambda} u(t)=e^{-\lambda t C} D_{t}^{\theta}\left(e^{\lambda t} u(t)\right)$ with $\theta \in(0,1)$ and $a, b, c$ are real constants. By using the principle of compressed mapping, spectral method, and stability analysis, the existence, uniqueness, structural stability, and numerical analysis of solutions were investigated.

In [19], by using a new mixed monotone operators fixed point theorem with perturbation, the authors studied the existence and uniqueness of positive solution for the following nonlinear fractional differential equation boundary value problem:

$$
\left\{\begin{array}{l}
D_{0^{+}}^{\alpha} u(t)=f(t, u(t), u(t))+g(t, u(t)), \quad t \in[0,1] \\
u(0)=u(1)=u^{\prime}(0)=u^{\prime}(1),
\end{array}\right.
$$

where $D_{0^{+}}^{\alpha}$ is the standard Riemann-Liouville fractional derivative of order $3<\alpha \leq$ $4, f(t, u, v):[0,1] \times[0,+\infty) \times[0,+\infty) \rightarrow \times[0,+\infty)$ is continuous, and $g(t, u):[0,1] \times$ $[0,+\infty) \rightarrow[0,+\infty)$ is continuous with $g(t, 0) \not \equiv$.

Based on the theory of $\mu_{0}$-positive linear operator and the Banach contraction map principle, Zhang and Zhong in [20] obtained the existence and uniqueness for the following fractional differential equation integral boundary value problem:

$$
\left\{\begin{array}{l}
D_{0^{+}}^{\alpha} z(t)+f(t, z(t))=0, \quad t \in[0,1] \\
z(0)=z^{\prime}(0)=\cdots=z^{n-2}(0)=0 \\
D_{0^{+}}^{\beta} z(1)=\lambda \int_{0}^{\eta} h(s) D_{0^{+}}^{\gamma} z(s) d s
\end{array}\right.
$$

where $n-1<\alpha \leq n(n \geq 3), \beta \geq 1, \alpha-\beta>1,0<\eta \leq 1, \lambda>0$ is a parameter, $D_{0^{+}}^{\alpha}$ is the Riemann-Liouville fractional derivative, $f(t, u, v):[0,1] \times \mathbb{R} \rightarrow \mathbb{R}$ is continuous and $g \in$ $L^{1}[0,1]$.

By means of the reducing method of fractional orders, the upper and lower solutions methods, and the Schauder fixed point theorem, Zhang, Liu, and Wu in [21] investigated the existence of positive solutions for the following fractional differential equations multipoint boundary value problem:

$$
\left\{\begin{array}{l}
-D_{0^{+}}^{\gamma} u(t)=\lambda f\left(u(t), D_{0^{+}}^{\mu_{1}} u(t), D_{0^{+}}^{\mu_{2}} u(t), \ldots, D_{0^{+}}^{\mu_{n-1}} u(t)\right), \quad t \in[0,1], \\
u(0)=0, \quad D_{0^{+}}^{\mu_{i}} u(0)=0, \quad 1 \leq i \leq n-2, \\
D_{0^{+}}^{\mu} u(1)=\sum_{j=1}^{p-2} a_{j} D_{0_{+}}^{\mu} u\left(\xi_{j}\right),
\end{array}\right.
$$

where $D_{0^{+}}^{\gamma}$ is the Riemann-Liouville fractional derivative, $\lambda>0$ is a parameter, $n-1<\gamma \leq$ $n, n-i-1 \leq \gamma-\mu_{i} \leq n-i(i=1,2, \ldots, n-2), \mu-\mu_{n-1}>0, \gamma-\mu_{n-1} \leq 2, \gamma-\mu>1, a_{j} \geq 0$ $(j=1,2, \ldots, p-2) 0<\xi_{1}<\xi_{2}<\cdots<1 ; f:(0,+\infty)^{n} \rightarrow R^{+}$is continuous. 
Inspired by the above-mentioned excellent works, we aim to investigate the existenceuniqueness of solutions for BVP (1.1) and BVP (1.2), respectively. As far as we know, the high-order Riemann-Liouville tempered fractional differential equations integral boundary values problems have seldom been researched up to now. The main features of the present paper are as follows. Firstly, the tempered fractional derivative ${ }_{0}^{R} \mathbb{D}_{t}^{\alpha, \lambda}$ is more general than the standard Riemann-Liouville fractional derivative ${ }_{0}^{R} D_{t}^{\alpha}$. For instance, let $\lambda=0$ and $g(t, u(t)) \equiv 0$, then BVP (1.1) reduces to the BVP in [22]; let $\lambda=0, b=0$, and $a(s) \equiv 0$, then BVP (1.2) is a special case in [20]. Secondly, the integral boundary conditions involving tempered fractional derivative are more general cases, which covers the common integral boundary conditions as special cases. For example, let $\lambda=0, a(t) \equiv 0, b(t) \equiv 1$, then the Riemann-Stieltjes integral boundary condition in (1.1) is reduced to a general integral boundary value condition. Finally, BVP (1.1) and BVP (1.2) are more general than those in the above-mentioned literature works. For instance, if $b=0,2<\alpha \leq 3$, and $\lambda=0$, BVP (1.2) presented in [23] has more special cases.

In this paper, it is not necessary for the operator to be completely continuous or compact, nor fix the existence of upper and lower solutions. For system (1.1), the existenceuniqueness and monotone iteration of positive solution are obtained by employing a class of sum-type operators fixed point theorems; for system (1.2), the existence and uniqueness of nontrivial solutions are investigated by means of a new fixed point theorem of increasing $\varphi-(h, \delta)$-concave operator, which is defined on a new set $P_{h, \delta}$. Furthermore, our conclusions can not only guarantee the existence-uniqueness of solutions, but also construct successive sequences for approximating the unique solution. In the end, it is worth mentioning that some important properties of the Green's function rely on the parameter $\lambda$.

The paper is organized as follows. In Sect. 2, we present some necessary definitions and preliminary results that will be used to prove our main results. In Sects. 3 and 4, we prove the main results about the existence-uniqueness of solutions for the Riemann-Stieltjes integral boundary value problems (1.1) and (1.2), respectively. Finally, two examples are given to illustrate the validity of our main results.

\section{Preliminaries}

A nonempty closed convex set $P \subset E$ is a cone if it satisfies:

$$
\begin{aligned}
& \left(I_{1}\right) x \in P, \lambda \geq 0 \Rightarrow \lambda x \in P ; \\
& \left(I_{2}\right) x \in P,-x \in P \Rightarrow x=\theta .
\end{aligned}
$$

Suppose that $(E,\|\cdot\|)$ is a real Banach space which is partially ordered by a cone $P \subset E$, that is, $x \leq y$ if and only if $y-x \in P$. If $x \leq y$ and $x \neq y$, then we denote $x<y$ or $y>x$. By $\theta$ we denote the zero element of $E$.

Definition 2.1 ([19]) $P$ is called normal if there exists $M>0$ such that, for all $x, y \in E$, $\theta \leq x \leq y$ implies $\|x\| \leq\|y\|$; in this case $M$ is the infimum of such constant, it is called normality constant of $P$.

In addition, for given $h>\theta$, we denote by $P_{h}$ the set $P_{h}=\{x \in E \mid x \sim h\}$, in which $\sim$ is an equivalence relation, i.e., $x \sim y$ means that there exist $\lambda>0$ and $\mu>0$ such that $\lambda x \geq y \geq \mu x$ for all $x, y \in E$. 
Definition 2.2 ([24]) An operator $A: P \times P \rightarrow P$ is said to be a mixed monotone operator if $A(x, y)$ is increasing in $x$ and decreasing in $y$, i.e., $u_{i}, v_{i}(i=1,2) \in P, u_{1}<u_{2}, v_{1}>v_{2}$ imply $A\left(u_{1}, v_{1}\right) \leq A\left(u_{2}, v_{2}\right)$. Element $x \in P$ is called a fixed point of $A$ if $A(x, x)=x$.

Definition 2.3 ([19]) $A: P \rightarrow P$ is said to be sub-homogeneous if it satisfies

$$
A(t x) \geq t A x, \quad \forall t \in(0,1), x \in P .
$$

Lemma $2.1([20])$ Let $h(t) \in C[0,1] \bigcap L^{1}[0,1], \alpha>0$, then

$$
{ }_{0} I_{t}^{\alpha R} D_{t}^{\alpha} h(t)=h(t)+c_{1} t^{\alpha-1}+c_{2} t^{\alpha-2}+\cdots+c_{n} t^{\alpha-n}
$$

where $c_{i} \in R, i=1,2,3, \ldots, n(n=[\alpha]+1)$.

Lemma $2.2([25])$

(1) If $u \in L^{1}(0,1), \alpha>\beta>0$, then

$$
{ }_{0} I_{t}^{\alpha}{ }_{0} I_{t}^{\beta} u(t)={ }_{0} I_{t}^{\alpha+\beta} u(t), \quad{ }_{0}^{R} D_{t}^{\beta}{ }_{0} I_{t}^{\alpha} u(t)={ }_{0} I_{t}^{\alpha-\beta}, \quad{ }_{0}^{R} D_{t}^{\beta} I_{t}^{\beta} u(t)=u(t) .
$$

(2) If $\rho>0, \mu>0$, then

$$
{ }_{0}^{R} D_{t}^{\rho} t^{\mu-1}=\frac{\Gamma(\mu)}{\Gamma(\mu-\rho)} t^{\mu-\rho-1}
$$

Lemma 2.3 Given $g \in C(0,1) \bigcap L^{1}(0,1), n-1<\alpha \leq n$, then the unique solution of

$$
\left\{\begin{array}{l}
{ }_{0}^{R} \mathbb{D}_{t}^{\alpha, \lambda} u(t)+g(t)=0, \quad t \in(0,1), \\
u(0)={ }_{0}^{R} \mathbb{D}_{t}^{\gamma_{1}, \lambda} u(0)={ }_{0}^{R} \mathbb{D}_{t}^{\gamma_{2}, \lambda} u(0)=\cdots={ }_{0}^{R} \mathbb{D}_{t}^{\gamma_{n-2}, \lambda} u(0)=0, \\
{ }_{0}^{R} \mathbb{D}_{t}^{\beta_{1}, \lambda} u(1)=\int_{0}^{\eta} b(s)_{0}^{R} \mathbb{D}_{t}^{\beta_{2}, \lambda} u(s) d A(s)+\int_{0}^{1} a(s){ }_{0}^{R} \mathbb{D}_{t}^{\beta_{3}, \lambda} u(s) d A(s)
\end{array}\right.
$$

is

$$
u(t)=\int_{0}^{1} G(t, s) g(s) d s, \quad t \in[0,1]
$$

where

$$
\begin{aligned}
G(t, s)= & G_{1}(t, s)+\frac{t^{\alpha-1} e^{-\lambda t}}{\Delta \Gamma\left(\alpha-\beta_{2}\right)} \int_{0}^{\eta} b(t) G_{2}(t, s) d A(t) \\
& +\frac{t^{\alpha-1} e^{-\lambda t}}{\Delta \Gamma\left(\alpha-\beta_{3}\right)} \int_{0}^{1} a(t) G_{3}(t, s) d A(t),
\end{aligned}
$$

in which

$$
\begin{aligned}
& \Delta=\frac{e^{-\lambda}}{\Gamma\left(\alpha-\beta_{1}\right)}-\frac{\delta_{1}}{\Gamma\left(\alpha-\beta_{2}\right)}-\frac{\delta_{2}}{\Gamma\left(\alpha-\beta_{3}\right)}, \\
& \delta_{1}=\int_{0}^{\eta} e^{-\lambda s} s^{\alpha-\beta_{2}-1} b(s) d A(s), \delta_{2}=\int_{0}^{1} e^{-\lambda s} s^{\alpha-\beta_{3}-1} a(s) d A(s),
\end{aligned}
$$




$$
\begin{aligned}
& G_{1}(t, s)=\frac{e^{\lambda(s-t)}}{\Gamma(\alpha)} \begin{cases}t^{\alpha-1}(1-s)^{\alpha-\beta_{1}-1}-(t-s)^{\alpha-1}, & 0 \leq s \leq t \leq 1, \\
t^{\alpha-1}(1-s)^{\alpha-\beta_{1}-1}, & 0 \leq t \leq s \leq 1,\end{cases} \\
& G_{2}(t, s)=\frac{e^{\lambda(s-t)}}{\Gamma(\alpha)} \begin{cases}t^{\alpha-\beta_{2}-1}(1-s)^{\alpha-\beta_{1}-1}-(t-s)^{\alpha-\beta_{2}-1}, & 0 \leq s \leq t \leq 1, \\
t^{\alpha-\beta_{2}-1}(1-s)^{\alpha-\beta_{1}-1}, & 0 \leq t \leq s \leq 1,\end{cases} \\
& G_{3}(t, s)=\frac{e^{\lambda(s-t)}}{\Gamma(\alpha)} \begin{cases}t^{\alpha-\beta_{3}-1}(1-s)^{\alpha-\beta_{1}-1}-(t-s)^{\alpha-\beta_{3}-1}, & 0 \leq s \leq t \leq 1, \\
t^{\alpha-\beta_{3}-1}(1-s)^{\alpha-\beta_{1}-1}, & 0 \leq t \leq s \leq 1 .\end{cases}
\end{aligned}
$$

Proof For system (2.1), by using Lemma 2.1, it is clear to see that

$$
e^{\lambda t} u(t)=-{ }_{0} I_{t}^{\alpha} e^{\lambda t} g(t)+c_{1} t^{\alpha-1}+c_{2} t^{\alpha-2}+\cdots+c_{n-1} t^{\alpha-(n-1)}+c_{n} t^{\alpha-n}
$$

From $u(0)=0$, we obtain $c_{n}=0$ and

$$
e^{\lambda t} u(t)=-{ }_{0} I_{t}^{\alpha} e^{\lambda t} g(t)+c_{1} t^{\alpha-1}+c_{2} t^{\alpha-2}+\cdots+c_{n-1} t^{\alpha-(n-1)} .
$$

Applying the Riemann-Liouville tempered fractional derivative operator ${ }_{0}^{R} \mathbb{D}_{t}^{\gamma_{1}, \lambda}$ on both sides of (2.5), and by Lemma 2.2, we can simply obtain

$$
\begin{aligned}
{ }_{0}^{R} \mathbb{D}_{t}^{\gamma_{1}, \lambda} u(t)= & { }_{0}^{R} \mathbb{D}_{t}^{\gamma_{1}, \lambda}\left\{-e^{-\lambda t}{ }_{0} I_{t}^{\alpha} e^{\lambda t} g(t)+e^{-\lambda t}\left(c_{1} t^{\alpha-1}+c_{2} t^{\alpha-2}+\cdots+c_{n-1} t^{\alpha-(n-1)}\right)\right\} \\
= & e_{0}^{-\lambda t R} D_{t}^{\gamma_{1}}\left\{-{ }_{0} I_{t}^{\alpha} e^{\lambda t} g(t)+c_{1} t^{\alpha-1}+c_{2} t^{\alpha-2}+\cdots+c_{n-1} t^{\alpha-(n-1)}\right\} \\
= & -e^{-\lambda t}{ }_{0} I_{t}^{\alpha-\gamma_{1}} e^{\lambda t} g(t)+c_{1} e^{-\lambda t R}{ }_{0}^{\gamma_{1}} t^{\alpha-1} \\
& +c_{2} e^{-\lambda t R} D_{t}^{\gamma_{1}} t^{\alpha-2}+\cdots+c_{n-1} e^{-\lambda t R}{ }_{0}^{\gamma_{1}} t_{t}^{\alpha-(n-1)} \\
= & \frac{-1}{\Gamma\left(\alpha-\gamma_{1}\right)} \int_{0}^{t}(t-s)^{\alpha-\gamma_{1}-1} e^{\lambda(s-t)} g(s) d s+c_{1} \frac{\Gamma(\alpha) e^{-\lambda t}}{\Gamma\left(\alpha-\gamma_{1}\right)} t^{\alpha-1-\gamma_{1}} \\
& +c_{2} \frac{\Gamma(\alpha-1) e^{-\lambda t}}{\Gamma\left(\alpha-1-\gamma_{1}\right)} t^{\alpha-2-\gamma_{1}}+\cdots+c_{n-1} \frac{\Gamma(\alpha-n+2) e^{-\lambda t}}{\Gamma\left(\alpha-n+2-\gamma_{1}\right)} t^{\alpha-n+1-\gamma_{1}} .
\end{aligned}
$$

From ${ }_{0}^{R} \mathbb{D}_{t}^{\gamma_{1}, \lambda} u(0)=0$ and $n-2 \leq \alpha-\gamma_{1} \leq n-1$, we obtain $c_{n-1}=0$. Similarly, we can deduce that $c_{n-2}=c_{n-3}=\cdots=c_{2}=0$, therefore, (2.5) can be reduced as follows:

$$
\begin{aligned}
u(t) & =-e^{-\lambda t}{ }_{0} I_{t}^{\alpha} e^{\lambda t} g(t)+c_{1} e^{-\lambda t} t^{\alpha-1} \\
& =-e^{-\lambda t} \int_{0}^{t} \frac{(t-s)^{\alpha-1} e^{\lambda s}}{\Gamma(\alpha)} g(s) d s+c_{1} e^{-\lambda t} t^{\alpha-1} .
\end{aligned}
$$

Furthermore, applying the Riemann-Liouville tempered fractional derivative operator ${ }_{0}^{R} \mathbb{D}_{t}^{\beta_{i}, \lambda}$ on both sides of (2.6), we obtain

$$
\begin{aligned}
{ }_{0}^{R} \mathbb{D}_{t}^{\beta_{i}, \lambda} u(t) & =-{ }_{0}^{R} \mathbb{D}_{t}^{\beta_{i}, \lambda}\left(e^{-\lambda t}{ }_{0} I_{t}^{\alpha} e^{\lambda t} g(t)\right)+c_{1}{ }_{0}^{R} \mathbb{D}_{t}^{\beta_{i}, \lambda}\left(e^{-\lambda t} t^{\alpha-1}\right) \\
& =-e^{-\lambda t R} D_{t}^{\beta_{i}}\left({ }_{0} I_{t}^{\alpha} e^{\lambda t} g(t)\right)+c_{1} e^{-\lambda t R} D_{t}^{\beta_{i}}\left(t^{\alpha-1}\right) \\
& =-e^{-\lambda t}{ }_{0} I_{t}^{\alpha-\beta_{i}}\left(e^{\lambda t} g(t)\right)+c_{1} \frac{\Gamma(\alpha)}{\Gamma\left(\alpha-\beta_{i}\right)} e^{-\lambda t} t^{\alpha-1-\beta_{i}} \\
& =-\int_{0}^{t} \frac{(t-s)^{\alpha-\beta_{i}-1} e^{\lambda(s-t)}}{\Gamma\left(\alpha-\beta_{i}\right)} g(s) d s+c_{1} \frac{\Gamma(\alpha)}{\Gamma\left(\alpha-\beta_{i}\right)} e^{-\lambda t} t^{\alpha-1-\beta_{i}} .
\end{aligned}
$$


From (2.7), we can know that

$$
\begin{aligned}
& { }_{0}^{R} \mathbb{D}_{t}^{\beta_{1}, \lambda} u(1)=-\int_{0}^{1} \frac{(1-s)^{\alpha-\beta_{1}-1} e^{\lambda(s-1)}}{\Gamma\left(\alpha-\beta_{1}\right)} g(s) d s+c_{1} \frac{\Gamma(\alpha)}{\Gamma\left(\alpha-\beta_{1}\right)} e^{-\lambda}, \\
& { }_{0}^{R} \mathbb{D}_{t}^{\beta_{2}, \lambda} u(t)=-\int_{0}^{t} \frac{(t-s)^{\alpha-\beta_{2}-1} e^{\lambda(s-t)}}{\Gamma\left(\alpha-\beta_{2}\right)} g(s) d s+c_{1} \frac{\Gamma(\alpha)}{\Gamma\left(\alpha-\beta_{2}\right)} e^{-\lambda t} t^{\alpha-1-\beta_{2}}, \\
& { }_{0}^{R} \mathbb{D}_{t}^{\beta_{3}, \lambda} u(t)=-\int_{0}^{t} \frac{(t-s)^{\alpha-\beta_{3}-1} e^{\lambda(s-t)}}{\Gamma\left(\alpha-\beta_{3}\right)} g(s) d s+c_{1} \frac{\Gamma(\alpha)}{\Gamma\left(\alpha-\beta_{3}\right)} e^{-\lambda t} t^{\alpha-1-\beta_{3}} .
\end{aligned}
$$

Substituting (2.8)-(2.10) into the integral boundary value conditions ${ }_{0}^{R} \mathbb{D}_{t}^{\beta_{1}, \lambda} u(1)=\int_{0}^{\eta} b(s) \times$ ${ }_{0}^{R} \mathbb{D}_{t}^{\beta_{2}, \lambda} u(s) d A(s)+\int_{0}^{1} a(s)_{0}^{R} \mathbb{D}_{t}^{\beta_{3}, \lambda} u(s) d A(s)$, we obtain

$$
\begin{aligned}
c_{1}= & (\Gamma(\alpha) \Delta)^{-1}\left\{\int_{0}^{1} \frac{(1-s)^{\alpha-\beta_{1}-1} e^{\lambda(s-1)}}{\Gamma\left(\alpha-\beta_{1}\right)} g(s) d s\right. \\
& -\int_{0}^{\eta} b(t) d A(t) \int_{0}^{t} \frac{(t-s)^{\alpha-\beta_{2}-1} e^{\lambda(s-t)}}{\Gamma\left(\alpha-\beta_{2}\right)} g(s) d s \\
& \left.-\int_{0}^{1} a(t) d A(t) \int_{0}^{t} \frac{(t-s)^{\alpha-\beta_{3}-1} e^{\lambda(s-t)}}{\Gamma\left(\alpha-\beta_{3}\right)} g(s) d s\right\} .
\end{aligned}
$$

At last, combining (2.11) with (2.6), we get

$$
\begin{aligned}
& u(t)=-e^{-\lambda t} \int_{0}^{t} \frac{(t-s)^{\alpha-1} e^{\lambda s}}{\Gamma(\alpha)} g(s) d s+\frac{e^{-\lambda t} t^{\alpha-1}}{\Gamma(\alpha) \Delta}\left\{\int_{0}^{1} \frac{(1-s)^{\alpha-\beta_{1}-1} e^{-\lambda}}{\Gamma\left(\alpha-\beta_{1}\right)} e^{\lambda s} g(s) d s\right. \\
& -\int_{0}^{\eta} b(t) d A(t) \int_{0}^{t} \frac{(t-s)^{\alpha-\beta_{2}-1} e^{\lambda(s-t)}}{\Gamma\left(\alpha-\beta_{2}\right)} g(s) d s \\
& \left.-\int_{0}^{1} a(t) d A(t) \int_{0}^{t} \frac{(t-s)^{\alpha-\beta_{3}-1} e^{\lambda(s-t)}}{\Gamma\left(\alpha-\beta_{3}\right)} g(s) d s\right\} \\
& =-e^{-\lambda t} \int_{0}^{t} \frac{(t-s)^{\alpha-1} e^{\lambda s}}{\Gamma(\alpha)} g(s) d s+\left(\frac{e^{-\lambda t} t^{\alpha-1}}{\Gamma(\alpha)}+\frac{e^{-\lambda t} t^{\alpha-1}}{\Gamma(\alpha) \Gamma\left(\alpha-\beta_{2}\right) \Delta} \delta_{1}\right. \\
& \left.+\frac{e^{-\lambda t} t^{\alpha-1}}{\Gamma(\alpha) \Gamma\left(\alpha-\beta_{3}\right) \Delta} \delta_{2}\right) \int_{0}^{1}(1-s)^{\alpha-\beta_{1}-1} e^{\lambda s} g(s) d s \\
& -\frac{e^{-\lambda t} t^{\alpha-1}}{\Gamma(\alpha) \Gamma\left(\alpha-\beta_{2}\right) \Delta} \int_{0}^{\eta} b(t) d A(t) \int_{0}^{t}(t-s)^{\alpha-\beta_{2}-1} e^{\lambda(s-t)} g(s) d s \\
& -\frac{e^{-\lambda t} t^{\alpha-1}}{\Gamma(\alpha) \Gamma\left(\alpha-\beta_{3}\right) \Delta} \int_{0}^{1} a(t) d A(t) \int_{0}^{t}(t-s)^{\alpha-\beta_{3}-1} e^{\lambda(s-t)} g(s) d s \\
& =-\int_{0}^{t} \frac{(t-s)^{\alpha-1} e^{-\lambda t} e^{\lambda s}}{\Gamma(\alpha)} g(s) d s+\int_{0}^{1} \frac{(1-s)^{\alpha-\beta_{1}-1} t^{\alpha-1} e^{\lambda(s-t)}}{\Gamma(\alpha)} g(s) d s \\
& +\frac{t^{\alpha-1} e^{-\lambda t}}{\Gamma\left(\alpha-\beta_{2}\right) \Delta} \int_{0}^{\eta} b(t) d A(t) \int_{0}^{1} \frac{(1-s)^{\alpha-\beta_{1}-1} t^{\alpha-\beta_{2}-1} e^{\lambda(s-t)}}{\Gamma(\alpha)} g(s) d s \\
& -\frac{t^{\alpha-1} e^{-\lambda t}}{\Gamma(\alpha) \Gamma\left(\alpha-\beta_{2}\right) \Delta} \int_{0}^{\eta} b(t) d A(t) \int_{0}^{t} \frac{(t-s)^{\alpha-\beta_{2}-1} e^{\lambda(s-t)}}{\Gamma(\alpha)} g(s) d s \\
& +\frac{t^{\alpha-1} e^{-\lambda t}}{\Gamma\left(\alpha-\beta_{3}\right) \Delta} \int_{0}^{1} a(t) d A(t) \int_{0}^{1} \frac{(1-s)^{\alpha-\beta_{1}-1} t^{\alpha-\beta_{3}-1} e^{\lambda(s-t)}}{\Gamma(\alpha)} g(s) d s \\
& -\frac{t^{\alpha-1} e^{-\lambda t}}{\Gamma\left(\alpha-\beta_{3}\right) \Delta} \int_{0}^{1} a(t) d A(t) \int_{0}^{t} \frac{(t-s)^{\alpha-\beta_{3}-1} e^{\lambda(s-t)}}{\Gamma(\alpha)} g(s) d s
\end{aligned}
$$




$$
\begin{aligned}
= & \int_{0}^{1} G_{1}(t, s) g(s) d s+\frac{t^{\alpha-1} e^{-\lambda t}}{\Gamma\left(\alpha-\beta_{2}\right) \Delta} \int_{0}^{1} g(s) d s \int_{0}^{\eta} G_{2}(t, s) b(t) d A(t) \\
& +\frac{t^{\alpha-1} e^{-\lambda t}}{\Gamma\left(\alpha-\beta_{3}\right) \Delta} \int_{0}^{1} g(s) d s \int_{0}^{1} G_{3}(t, s) a(t) d A(t) \\
= & \int_{0}^{1} G(t, s) g(s) d s,
\end{aligned}
$$

where

$$
\begin{aligned}
G(t, s)= & G_{1}(t, s)+\frac{t^{\alpha-1} e^{-\lambda t}}{\Delta \Gamma\left(\alpha-\beta_{2}\right)} \int_{0}^{\eta} b(t) G_{2}(t, s) d A(t) \\
& +\frac{t^{\alpha-1} e^{-\lambda t}}{\Delta \Gamma\left(\alpha-\beta_{3}\right)} \int_{0}^{1} a(t) G_{3}(t, s) d A(t)
\end{aligned}
$$

is the Green function of system (2.1). Then, we complete the proof.

\section{Lemma 2.4 Suppose that}

$$
\text { (H) } \frac{\Gamma\left(\alpha-\beta_{1}\right) e^{\lambda}}{\Gamma\left(\alpha-\beta_{2}\right)} \delta_{1}+\frac{\Gamma\left(\alpha-\beta_{1}\right) e^{\lambda}}{\Gamma\left(\alpha-\beta_{3}\right)} \delta_{2}<1,
$$

then, for all $t, s \in(0,1)$, the Green function $G(t, s)$ defined by (2.3) satisfies

$\left(A_{1}\right) G_{1}(t, s)>0, G_{2}(t, s)>0, G_{3}(t, s)>0$, and $G(t, s)>0$;

$\left(A_{2}\right) \frac{e^{\lambda s}\left[(1-s)^{\alpha-\beta_{1}-1}-(1-s)^{\alpha-1}\right]}{\Gamma(\alpha)} e^{-\lambda t} t^{\alpha-1} \leq G_{1}(t, s) \leq \frac{e^{\lambda s}(1-s)^{\alpha-\beta_{1}-1}}{\Gamma(\alpha)} e^{-\lambda t} t^{\alpha-1}$;

$\left(A_{3}\right) \frac{e^{\lambda s}\left[(1-s)^{\alpha-\beta_{1}-1}-(1-s)^{\alpha-\beta_{2}-1}\right]}{\Gamma(\alpha)} e^{-\lambda t} t^{\alpha-\beta_{2}-1} \leq G_{2}(t, s) \leq \frac{e^{\lambda s}(1-s)^{\alpha-\beta_{1}-1}}{\Gamma(\alpha)} e^{-\lambda t} t^{\alpha-\beta_{2}-1}$;

$\left(A_{4}\right) \frac{e^{\lambda s}\left[(1-s)^{\alpha-\beta_{1}-1}-(1-s)^{\alpha-\beta_{3}-1}\right]}{\Gamma(\alpha)} e^{-\lambda t} t^{\alpha-\beta_{3}-1} \leq G_{3}(t, s) \leq \frac{e^{\lambda s}(1-s)^{\alpha-\beta_{1}-1}}{\Gamma(\alpha)} e^{-\lambda t} t^{\alpha-\beta_{3}-1}$;

$\left(A_{5}\right) m(s) e^{-\lambda t} t^{\alpha-1} \leq G(t, s) \leq M(s) e^{-\lambda t} t^{\alpha-1}$, where

$$
M(s)=\left(\frac{1}{\Gamma(\alpha)}+\frac{\delta_{1}}{\Delta \Gamma(\alpha) \Gamma\left(\alpha-\beta_{2}\right)}+\frac{\delta_{2}}{\Delta \Gamma(\alpha) \Gamma\left(\alpha-\beta_{3}\right)}\right) e^{\lambda s}(1-s)^{\alpha-\beta_{1}-1}
$$

and

$$
\begin{aligned}
m(s)= & \left(\frac{(1-s)^{\alpha-\beta_{1}-1}-(1-s)^{\alpha-1}}{\Gamma(\alpha)}+\frac{(1-s)^{\alpha-\beta_{1}-1}-(1-s)^{\alpha-\beta_{2}-1}}{\Delta \Gamma(\alpha) \Gamma\left(\alpha-\beta_{2}\right)} \delta_{1}\right. \\
& \left.+\frac{(1-s)^{\alpha-\beta_{1}-1}-(1-s)^{\alpha-\beta_{3}-1}}{\Delta \Gamma(\alpha) \Gamma\left(\alpha-\beta_{3}\right)} \delta_{2}\right) e^{\lambda s} .
\end{aligned}
$$

Proof From condition $(H)$, we can get $\Delta>0$. In addition, for all $G_{i}(t, s)(i=1,2,3)$ and $\forall t, s \in(0,1)$, the right inequality of $\left(A_{2}\right),\left(A_{3}\right)$, and $\left(A_{4}\right)$ holds. Now, we only need to prove the left inequality.

If $0 \leq s \leq t \leq 1$, then we have $0 \leq t-s \leq t-t s=(1-s) t$, and thus $(t-s)^{\alpha-1} \leq(1-s)^{\alpha-1} t^{\alpha-1}$. Therefore, we can know that

$$
\begin{aligned}
G_{1}(t, s) & =\frac{e^{\lambda(s-t)}}{\Gamma(\alpha)}\left[t^{\alpha-1}(1-s)^{\alpha-\beta_{1}-1}-(t-s)^{\alpha-1}\right] \\
& \geq \frac{e^{\lambda(s-t)}}{\Gamma(\alpha)}\left[t^{\alpha-1}(1-s)^{\alpha-\beta_{1}-1}-(1-s)^{\alpha-1} t^{\alpha-1}\right] \\
& =\frac{e^{\lambda s}\left[(1-s)^{\alpha-\beta_{1}-1}-(1-s)^{\alpha-1}\right]}{\Gamma(\alpha)} e^{-\lambda t} t^{\alpha-1} .
\end{aligned}
$$


If $0 \leq t \leq s \leq 1$,

$$
\begin{aligned}
G_{1}(t, s) & =\frac{e^{\lambda(s-t)}}{\Gamma(\alpha)} t^{\alpha-1}(1-s)^{\alpha-\beta_{1}-1} \\
& \geq \frac{e^{\lambda s}\left[(1-s)^{\alpha-\beta_{1}-1}-(1-s)^{\alpha-1}\right]}{\Gamma(\alpha)} e^{-\lambda t} t^{\alpha-1} .
\end{aligned}
$$

Also, because of $(1-s)^{\alpha-\beta_{1}-1}>(1-s)^{\alpha-1}$, we get $G_{1}(t, s) \geq 0$. In the same way, the similar conclusions can be obtained for $G_{2}(t, s)$ and $G_{3}(t, s)$. Finally, from $\left(A_{2}\right),\left(A_{3}\right)$, and $\left(A_{4}\right)$, conclusion $\left(A_{5}\right)$ holds and $G(t, s) \geq 0$. Hence, the proof is complete.

Lemma 2.5 ([19]) Let $\alpha \in(0,1), A: P \times P \rightarrow P$ be a mixed monotone operator and satisfy

$$
A\left(t x, t^{-1} y\right) \geq t^{\alpha} A(x, y), \quad t \in(0,1), x, y \in P .
$$

$B: P \rightarrow P$ is an increasing sub-homogeneous operator. Assume that

(I) there is $h_{0} \in P_{h}$ such that $A\left(h_{0}, h_{0}\right) \in P_{h}$ and $B h_{0} \in P_{h}$;

(II) there exists a constant $\delta_{0}>0$ such that $A(x, y) \geq \delta_{0} B x, \forall x, y \in P$.

Then

(1) $A: P_{h} \times P_{h} \rightarrow P_{h}, B: P_{h} \rightarrow P_{h}$;

(2) there exist $u_{0}, v_{0} \in P_{h}$ and $r \in(0,1)$ such that

$$
r v_{0} \leq u_{0}<v_{0}, \quad u_{0} \leq A\left(u_{0}, v_{0}\right)+B u_{0} \leq A\left(v_{0}, u_{0}\right)+B\left(v_{0}\right) \leq v_{0}
$$

(3) the operator equation $A(x, x)+B x=x$ has a unique solution $x^{*}$ in $P_{h}$;

(4) for any initial values $x_{0}, y_{0} \in P_{h}$, constructing successively the sequences

$$
x_{n}=A\left(x_{n-1}, y_{n-1}\right)+B x_{n-1}, \quad y_{n}=A\left(y_{n-1}, x_{n-1}\right)+B y_{n-1}, \quad n=1,2, \ldots,
$$

we have $x_{n} \rightarrow x^{*}$ and $y_{n} \rightarrow x^{*}$ as $n \rightarrow \infty$.

\section{Existence-uniqueness of positive solution for BVP (1.1)}

In this section, we consider the Banach space $C[0,1]$, the space of all continuous functions on $[0,1]$. It is evident that this space can be equipped with a partial order

$$
x, y \in C[0,1], \quad x \leq y \quad \Longleftrightarrow \quad x(t) \leq y(t) \quad \text { for } t \in[0,1]
$$

Setting $P=\{x \in C[0,1] \mid x(t) \geq 0, t \in[0,1]\}$ and $h(t)=e^{-\lambda t} t^{\alpha-1}$, we know that $P$ is a normal cone in $C[0,1]$.

Theorem 3.1 Assume that condition $(H)$ holds and

$\left(H_{1}\right) f(t, u, v):[0,1] \times[0,+\infty) \times[0,+\infty) \rightarrow[0,+\infty)$ is continuous, $g(t, u):[0,1] \times$ $[0,+\infty) \rightarrow[0,+\infty)$ is continuous with $g(t, 0) \not \equiv 0, a(t), b(t):[0,1] \rightarrow R^{+}$are continuous;

$\left(H_{2}\right) f(t, u, v)$ is increasing in $u \in[0,+\infty)$ for fixed $t \in[0,1]$ and $v \in[0,+\infty)$, decreasing in $v \in[0,+\infty)$ for fixed $t \in[0,1]$ and $u \in[0,+\infty)$, and $g(t, u)$ is increasing in $u \in$ $[0,+\infty)$ for fixed $t \in[0,1]$ 
$\left(H_{3}\right)$ for $\forall t \in[0,1], \gamma \in(0,1), u, v \in[0,+\infty)$, there exists a constant $\xi \in(0,1)$ such that $f\left(t, \gamma u, \gamma^{-1} v\right) \geq \gamma^{\xi} f(t, u, v)$ and $g(t, \gamma u) \geq \gamma g(t, u) ;$

$\left(H_{4}\right)$ for $\forall t \in[0,1]$ and $u, v \in[0,+\infty)$, there exists a constant $\delta_{0}>0$ such that $f(t, u, v) \geq$ $\delta_{0} g(t, u)$.

Then we have:

(I) there exist $u_{0}, v_{0} \in P_{h}$ and $r \in(0,1)$ such that $r v_{0} \leq u_{0}<v_{0}$ and

$$
\begin{aligned}
& u_{0}(t) \leq \int_{0}^{1} G(t, s) f\left(s, u_{0}(s), v_{0}(s)\right) d s+\int_{0}^{1} G(t, s) g\left(s, u_{0}(s)\right) d s, \quad t \in[0,1] \\
& v_{0}(t) \geq \int_{0}^{1} G(t, s) f\left(s, v_{0}(s), u_{0}(s)\right) d s+\int_{0}^{1} G(t, s) g\left(s, v_{0}(s)\right) d s, \quad t \in[0,1]
\end{aligned}
$$

where $h(t)=e^{-\lambda t} t^{\alpha-1}, t \in[0,1] ;$

(II) $B V P(1.1)$ has a unique positive solution $u^{*} \in P_{h}$;

(III) for any initial values $x_{0}, y_{0} \in P_{h}$, making successively the sequences

$$
\begin{aligned}
& x_{n}=\int_{0}^{1} G(t, s) f\left(s, x_{n-1}(s), y_{n-1}(s)\right) d s+\int_{0}^{1} G(t, s) g\left(s, x_{n-1}(s)\right) d s, \\
& y_{n}=\int_{0}^{1} G(t, s) f\left(s, y_{n-1}(s), x_{n-1}(s)\right) d s+\int_{0}^{1} G(t, s) g\left(s, y_{n-1}(s)\right) d s,
\end{aligned}
$$

we obtain $x_{n} \rightarrow u^{*}$ and $y_{n} \rightarrow u^{*}$ as $n \rightarrow \infty$.

Proof To begin with, from Lemma 2.3, BVP (1.1) has an integral formulation given by

$$
u(t)=\int_{0}^{1} G(t, s)[f(s, u(s), u(s))+g(s, u(s))] d s
$$

where $G(t, s)$ is given as in (2.3). Define two operators $A: P \times P \rightarrow E$ and $B: P \rightarrow E$ by

$$
A(u, v)(t)=\int_{0}^{1} G(t, s) f(s, u(s), v(s)) d s,(B u)(t)=\int_{0}^{1} G(t, s) g(s, u(s)) d s
$$

Evidently, $u$ is a solution of BVP (1.1) if and only if $u=A(u, u)+B(u)$. From $\left(H_{1}\right)$ and $\left(H_{2}\right)$, it is easy to know that $A: P \times P \rightarrow P$ is a mixed monotone operator and $B: P \rightarrow P$ is an increasing operator. Again, from $\left(H_{3}\right)$, for $\forall \gamma \in(0,1)$ and $u, v \in P$, we obtain

$$
\begin{aligned}
A\left(\gamma u, \gamma^{-1} v\right)(t) & =\int_{0}^{1} G(t, s) f\left(s, \gamma u(s), \gamma^{-1} v(s)\right) d s \\
& \geq \gamma^{\xi} \int_{0}^{1} G(t, s) f(s, u(s), v(s)) d s \\
& =\gamma^{\xi} A(u, v)(t) .
\end{aligned}
$$

That is, $A\left(\gamma u, \gamma^{-1} v\right) \geq \gamma^{\xi} A(u, v)$ for $\forall \gamma \in(0,1)$ and $u, v \in P$. Hence, the operator $A$ satisfies condition (2.12) in Lemma 2.5. In addition, for any $u \in P$ and $\gamma \in(0,1)$, from $\left(H_{3}\right)$ we know 
that

$$
B(\gamma u)(t)=\int_{0}^{1} G(t, s) g(s, \gamma u(s)) d s \geq \gamma \int_{0}^{1} G(t, s) g(s, u(s)) d s=\gamma B u(t),
$$

so the operator $B$ is a sub-homogeneous operator.

Next, since $h \in P_{h}$, setting $h_{0}=h$, we show that $A(h, h) \in P_{h}$ and $B h \in P_{h}$. From Lemma 2.3 and Lemma 2.4, we have

$$
\begin{aligned}
A(h, h)(t) & =\int_{0}^{1} G(t, s) f(s, h(s), h(s)) d s \leq \int_{0}^{1} M(s) e^{-\lambda t} t^{\alpha-1} f(s, h(s), h(s)) d s \\
& \leq \int_{0}^{1} M(s) e^{-\lambda t} t^{\alpha-1} f\left(s, h_{\max }, 0\right) d s \\
& =\int_{0}^{1} M(s) f\left(s, h_{\max }, 0\right) d s \cdot h(t)
\end{aligned}
$$

and

$$
\begin{aligned}
A(h, h)(t) & =\int_{0}^{1} G(t, s) f(s, h(s), h(s)) d s \geq \int_{0}^{1} m(s) e^{-\lambda t} t^{\alpha-1} f(s, h(s), h(s)) d s \\
& \geq \int_{0}^{1} m(s) e^{-\lambda t} t^{\alpha-1} f\left(s, 0, h_{\max }\right) d s \\
& =\int_{0}^{1} m(s) f\left(s, 0, h_{\max }\right) d s \cdot h(t),
\end{aligned}
$$

where $h_{\max }=\max \{h(t): t \in[0,1]\}$. Letting $L=\int_{0}^{1} M(s) f\left(s, h_{\max }, 0\right) d s$ and $l=\int_{0}^{1} m(s) f(s, 0$, $\left.h_{\max }\right) d s$. It is evident that $L>l>0$, we get $l h(t) \leq A(h, h) \leq L h(t)$, that is, $A(h, h) \in P_{h}$. Similarly, from

$$
\int_{0}^{1} m(s) g(s, 0) d s \cdot h(t) \leq(B h)(t) \leq \int_{0}^{1} M(s) g\left(s, h_{\max }\right) d s \cdot h(t)
$$

we obtain $B h \in P_{h}$. Then, condition $\left(I_{1}\right)$ of Lemma 2.5 is satisfied.

Finally, we show that condition $\left(I_{2}\right)$ of Lemma 2.5 is also satisfied. For $\forall u, v \in P$, from (3.1) and $\left(H_{4}\right)$, we get

$$
A(u, v)(t)=\int_{0}^{1} G(t, s) f(s, u(s), v(s)) d s \geq \delta_{0} \int_{0}^{1} G(t, s) g(s, u(s)) d s=\delta_{0} B u(t) .
$$

That is, $A(u, v) \geq \delta_{0} B u$ for $\forall u, v \in P_{h}$. Then, the conclusion of Theorem 3.1 follows Lemma 2.5 .

Corollary 3.1 Assume that condition $(H)$ holds and

$\left(H_{1}^{\prime}\right) f(t, u, v):[0,1] \times[0,+\infty) \times[0,+\infty) \rightarrow[0,+\infty)$ is a continuous function with $f\left(t, 0, h_{\max }\right) \not \equiv 0, a(t), b(t):[0,1] \rightarrow R^{+}$are continuous;

$\left(H_{2}^{\prime}\right) f(t, u, v)$ is increasing in $u \in[0,+\infty)$ for fixed $t \in[0,1]$ and $v \in[0,+\infty)$, decreasing in $v \in[0,+\infty)$ for fixed $t \in[0,1]$ and $u \in[0,+\infty)$;

$\left(H_{3}^{\prime}\right)$ for $\forall t \in[0,1], \gamma \in(0,1), u, v \in[0,+\infty)$, there exists a constant $\xi \in(0,1)$ such that $f\left(t, \gamma u, \gamma^{-1} v\right) \geq \gamma^{\xi} f(t, u, v)$. 
Then we have:

(I) the boundary value problem

$$
\left\{\begin{array}{l}
{ }_{0}^{R} \mathbb{D}_{t}^{\alpha, \lambda} u(t)+f(t, u(t), u(t))=0, \quad t \in(0,1), \\
u(0)={ }_{0}^{R} \mathbb{D}_{t}^{\gamma_{1}, \lambda} u(0)={ }_{0}^{R} \mathbb{D}_{t}^{\gamma_{2}, \lambda} u(0)=\cdots={ }_{0}^{R} \mathbb{D}_{t}^{\gamma_{n-2}, \lambda} u(0)=0, \\
{ }_{0}^{R} \mathbb{D}_{t}^{\beta_{1}, \lambda} u(1)=\int_{0}^{\eta} b(s){ }_{0}^{R} \mathbb{D}_{t}^{\beta_{2}, \lambda} u(s) d A(s)+\int_{0}^{1} a(s)_{0}^{R} \mathbb{D}_{t}^{\beta_{3}, \lambda} u(s) d A(s)
\end{array}\right.
$$

has a unique positive solution $u^{*} \in P_{h}$;

(II) there exist $u_{0}, v_{0} \in P_{h}$ and $r \in(0,1)$ such that $r v_{0} \leq u_{0}<v_{0}$ and

$$
\begin{aligned}
& u_{0}(t) \leq \int_{0}^{1} G(t, s) f\left(s, u_{0}(s), v_{0}(s)\right) d s, \\
& v_{0}(t) \geq \int_{0}^{1} G(t, s) f\left(s, v_{0}(s), u_{0}(s)\right) d s
\end{aligned}
$$

where $h(t)=e^{-\lambda t} t^{\alpha-1}, t \in[0,1]$;

(III) for any initial values $x_{0}, y_{0} \in P_{h}$, making successively the sequences

$$
\begin{gathered}
x_{n}=\int_{0}^{1} G(t, s) f\left(s, x_{n-1}(s), y_{n-1}(s)\right) d s, \\
y_{n}=\int_{0}^{1} G(t, s) f\left(s, y_{n-1}(s), x_{n-1}(s)\right) d s, \\
\text { we obtain } x_{n} \rightarrow u^{*} \text { and } y_{n} \rightarrow u^{*} \text { as } n \rightarrow \infty .
\end{gathered}
$$

Proof Let $g(t, u(t)) \equiv 0$, from Theorem 3.1, we get the conclusions.

\section{Existence-uniqueness of nontrivial solution for BVP (1.2)}

In this section, for $h>\theta$, taking another $\sigma \in P$ with $\theta \leq \sigma \leq h$, we define a new set $P_{h, \sigma}=\left\{x \in E \mid x+\sigma \in P_{h}\right\}$. Then we can see that $h \in P_{h, \sigma}$ and $P_{h, \sigma}=\{x \in E \mid$ there exist $\mu>$ 0 and $v>0$ such that $\mu h \leq x+\sigma \leq v h\}$. If $\sigma=\theta$, then $P_{h, \sigma}=P_{h}$.

Remark 4.1 $P_{h} \subseteq P_{h, \sigma}$ and $P_{h, \sigma}$ is not a subset of $P$ for some $\sigma, P_{h}$ and $P_{h, \sigma}$ are different two sets.

Definition 4.1 ([23]) Let $A: P_{h, \sigma} \rightarrow E$ be a given operator. For any $x \in P_{h, \sigma}$ and $t \in(0,1)$, there exist $\varphi(t)>t$ such that

$$
A(t x+(t-1) \sigma) \geq \varphi(t) A x+(\varphi(t)-1) \sigma
$$

Then $A$ is called a $\varphi-(h, \sigma)$-concave operator.

Lemma 4.1 ([23]) Let $P$ be normal and $A$ be an increasing $\varphi-(h, \sigma)$-concave operator with $A h \in P_{h, \sigma}$. Then $A$ has a unique fixed point $x^{*}$ in $P_{h, \sigma}$. Moreover, making the sequence $\omega_{n}=A \omega_{n-1}, n=1,2, \ldots$, we obtain $\left\|\omega_{n}-x^{*}\right\| \rightarrow 0$ as $n \rightarrow \infty$. 
Now, we define a new function $\tilde{h}(t)$ by $\tilde{h}(t): \triangleq \rho e^{-\lambda t} t^{\alpha-1}$, where $\rho>0$ is a constant and

$$
\begin{aligned}
\rho \geq & c \int_{0}^{1}\left(\frac{1}{\Gamma(\alpha)}+\frac{\delta_{1}}{\Delta \Gamma(\alpha) \Gamma\left(\alpha-\beta_{2}\right)}\right) e^{\lambda s}(1-s)^{\alpha-\beta_{1}-1} d s \\
& +c \int_{0}^{1} \frac{\delta_{2}}{\Delta \Gamma(\alpha) \Gamma\left(\alpha-\beta_{3}\right)} e^{\lambda s}(1-s)^{\alpha-\beta_{1}-1} d s .
\end{aligned}
$$

In addition, setting

$$
\begin{aligned}
\sigma(t): \triangleq & c \int_{0}^{1} G_{1}(t, s) d s+c \cdot \frac{t^{\alpha-1} e^{-\lambda t}}{\Delta \Gamma\left(\alpha-\beta_{2}\right)} \int_{0}^{1} d s \int_{0}^{\eta} b(t) G_{2}(t, s) d A(t) \\
& +c \cdot \frac{t^{\alpha-1} e^{-\lambda t}}{\Delta \Gamma\left(\alpha-\beta_{3}\right)} \int_{0}^{1} d s \int_{0}^{1} a(t) G_{3}(t, s) d A(t)
\end{aligned}
$$

from (4.3) and Lemma 2.4, we can know that $\sigma(t) \geq c e^{-\lambda t} t^{\alpha-1} \int_{0}^{1} m(s) d s \geq 0$ for $\forall t \in[0,1]$, so we get $\sigma \in P$. Furthermore, from (4.2) we have

$$
\begin{aligned}
\sigma(t) \leq & \left\{c \int_{0}^{1}\left[\frac{1}{\Gamma(\alpha)}+\frac{\delta_{1}}{\Delta \Gamma(\alpha) \Gamma\left(\alpha-\beta_{2}\right)}\right] e^{\lambda s}(1-s)^{\alpha-\beta_{1}-1} d s\right. \\
& \left.+c \int_{0}^{1} \frac{\delta_{2}}{\Delta \Gamma(\alpha) \Gamma\left(\alpha-\beta_{3}\right)} e^{\lambda s}(1-s)^{\alpha-\beta_{1}-1} d s\right\} e^{-\lambda t} t^{\alpha-1} \\
\leq & \rho e^{-\lambda t} t^{\alpha-1}=\tilde{h}(t) .
\end{aligned}
$$

Hence, it is easy to know that $0 \leq \sigma(t) \leq \tilde{h}(t)$ and $P_{\tilde{h}, \sigma}=\left\{u \in C[0,1] \mid u+\sigma \in P_{\tilde{h}}\right\}$

Theorem 4.1 Suppose that condition $(H)$ holds, $c>0$ and

$\left(H_{5}\right) \psi:[0,1] \times\left[-\sigma^{*},+\infty\right) \rightarrow(-\infty,+\infty)$ is increasing with respect to the second variable, where $\sigma^{*}=\max \{\sigma(t) \mid t \in[0,1]\}, a(t), b(t):[0,1] \rightarrow R^{+}$are continuous;

$\left(H_{6}\right)$ for any $\gamma \in[0,1]$, there exist $\varphi(\gamma)>\gamma$ such that

$$
\psi(t, \gamma x+(\gamma-1) y) \geq \varphi(\gamma) \psi(t, x), \quad \forall t \in[0,1], x \in(-\infty,+\infty), y \in\left[0, \sigma^{*}\right]
$$

$\left(H_{7}\right) \psi(t, 0) \geq 0$ with $\psi(t, 0) \not \equiv 0$ for $t \in[0,1]$.

Then, BVP (1.2) has a unique nontrivial solution $u^{*}$ in $P_{\tilde{h}, \sigma}$. Moreover, for any given $\omega_{0} \in$ $P_{\tilde{h}, \sigma}$, constructing successively the sequences

$$
\begin{aligned}
\omega_{n}= & \int_{0}^{1} G(t, s) \psi\left(s, \omega_{n-1}(s)\right) d s-c \int_{0}^{1} G_{1}(t, s) d s \\
& -\frac{c t^{\alpha-1} e^{-\lambda t}}{\Delta \Gamma\left(\alpha-\beta_{2}\right)} \int_{0}^{1} d s \int_{0}^{\eta} b(t) G_{2}(t, s) d A(t) \\
& -\frac{c b t^{\alpha-1} e^{-\lambda t}}{\Delta \Gamma\left(\alpha-\beta_{3}\right)} \int_{0}^{1} d s \int_{0}^{1} a(t) G_{3}(t, s) d A(t),
\end{aligned}
$$

we have $\omega_{n}(t) \rightarrow u^{*}(t)$ as $n \rightarrow \infty$. 
Proof Firstly, from Lemma 2.3, we can know that BVP (1.2) is equivalent to

$$
\begin{aligned}
u(t)= & \int_{0}^{1} G(t, s) f(s, u(s)) d s-c \int_{0}^{1} G(t, s) d s \\
= & \int_{0}^{1} G(t, s) f(s, u(s)) d s-c \int_{0}^{1} G_{1}(t, s) d s \\
& -c \cdot \frac{t^{\alpha-1} e^{-\lambda t}}{\Delta \Gamma\left(\alpha-\beta_{2}\right)} \int_{0}^{1} d s \int_{0}^{\eta} b(t) G_{2}(t, s) d A(t) \\
& -c \cdot \frac{t^{\alpha-1} e^{-\lambda t}}{\Delta \Gamma\left(\alpha-\beta_{3}\right)} \int_{0}^{1} d s \int_{0}^{1} a(t) G_{3}(t, s) d A(t) \\
= & \int_{0}^{1} G(t, s) f(s, u(s)) d s-\sigma(t) .
\end{aligned}
$$

Furthermore, for any $u \in P_{\tilde{h}, \sigma}$, let

$$
\Phi u(t)=\int_{0}^{1} G(t, s) \psi(s, u(s)) d s-\sigma(t), \quad t \in[0,1]
$$

Then, $u$ is a solution of BVP (1.2) if and only if $\Phi u=u$.

Secondly, we prove that $\Phi: P_{\tilde{h}, \sigma} \rightarrow E$ is an increasing $\varphi-(\tilde{h}, \sigma)$-concave operator. For $\forall u \in P_{\tilde{h}, \sigma}$ and $\gamma \in(0,1)$, from $\left(H_{6}\right)$ we have

$$
\begin{aligned}
\Phi(\gamma u+(\gamma-1) \sigma)(t) & =\int_{0}^{1} G(t, s) \psi(s, \gamma u(s)+(\gamma-1) \sigma(s)) d s-\sigma(t) \\
& \geq \varphi(\gamma) \int_{0}^{1} G(t, s) \psi(s, u(s)) d s-\sigma(t) \\
& =\varphi(\gamma)\left[\int_{0}^{1} G(t, s) \psi(s, u(s)) d s-\sigma(t)\right]+[\varphi(\gamma)-1] \sigma(t) \\
& =\varphi(\gamma) \Phi u(t)+(\varphi(\gamma)-1) \sigma(t),
\end{aligned}
$$

that is,

$$
\Phi(\gamma u+(\gamma-1) \sigma) \geq \varphi(\gamma) \Phi u+(\varphi(\gamma)-1) \sigma \quad \text { for } \forall \gamma \in(0,1) \text { and } u \in P_{\tilde{h}, \sigma} .
$$

In addition, for $\forall u_{1}, u_{2} \in P_{h, \sigma}$, and $u_{1}>u_{2}$, from the definition of $P_{\tilde{h}, \sigma}$, we have $u_{i}+\sigma \in P_{\tilde{h}}$, where $i=1,2$, so there exists $\mu_{i}>0$ such that $u_{i}(t)+\sigma(t) \geq \mu_{i} h(t)$ for $\forall t \in[0,1]$ and $i=1,2$, that is, $u_{i}(t) \geq \mu_{i} h(t)-\sigma(t) \geq-\sigma(t) \geq-\sigma^{*}(t)$. Hence, from Lemma 2.4, $\left(H_{5}\right)$, and (4.5), we know that

$$
\begin{aligned}
\Phi u_{1}(t)-\Phi u_{2}(t) & =\int_{0}^{1} G(t, s) \psi\left(s, u_{1}(s)\right) d s-\int_{0}^{1} G(t, s) \psi\left(s, u_{2}(s)\right) d s \\
& =\int_{0}^{1} G(t, s)\left[\psi\left(s, u_{1}(s)\right)-\psi\left(s, u_{2}(s)\right)\right] d s \\
& >0
\end{aligned}
$$

that is, $\Phi u_{1}>\Phi u_{2}$ for $\forall t \in[0,1]$. Evidently, $\Phi: P_{\tilde{h}, \sigma} \rightarrow E$ is an increasing $\varphi-(\tilde{h}, \sigma)$-concave operator. 
Finally, we show that $\Phi \tilde{h} \in P_{\tilde{h}, \sigma}$. From $\left(H_{5}\right),(4.5)$, and Lemma 2.4, we obtain

$$
\begin{aligned}
\Phi \tilde{h}(t)+\sigma(t) & =\int_{0}^{1} G(t, s) \psi(s, \tilde{h}(s)) d s=\int_{0}^{1} G(t, s) \psi\left(s, \rho e^{-\lambda t} s^{\alpha-1}\right) d s \\
& \leq \int_{0}^{1} M(s) e^{-\lambda t} t^{\alpha-1} \psi\left(s, \rho s^{\alpha-1}\right) d s \\
& =\frac{1}{\rho} \int_{0}^{1} M(s) \psi\left(s, \rho s^{\alpha-1}\right) d s \cdot \tilde{h}(t)
\end{aligned}
$$

and

$$
\begin{aligned}
\Phi \tilde{h}(t)+\sigma(t) & =\int_{0}^{1} G(t, s) \psi(s, \tilde{h}(s)) d s=\int_{0}^{1} G(t, s) \psi\left(s, \rho e^{-\lambda t} s^{\alpha-1}\right) d s \\
& \geq \int_{0}^{1} m(s) e^{-\lambda t} t^{\alpha-1} \psi(s, 0) d s \\
& =\frac{1}{\rho} \int_{0}^{1} m(s) \psi(s, 0) d s \cdot \tilde{h}(t) .
\end{aligned}
$$

Let

$$
N=\frac{1}{\rho} \int_{0}^{1} M(s) \psi\left(s, \rho s^{\alpha-1}\right) d s, \quad n=\frac{1}{\rho} \int_{0}^{1} m(s) \psi(s, 0) d s
$$

It is evident that $N \geq n>0$ and $n \tilde{h} \leq \Phi \tilde{h}+\sigma \leq N \tilde{h}$, that is, $\Phi \tilde{h} \in P_{\tilde{h}, \sigma}$.

So far, we have verified that all the conditions in Lemma 4.1 are satisfied, hence, the operator $\Phi$ has a unique fixed point $u^{*}$ in $P_{\tilde{h}, \sigma}$. Moreover, for any $\omega_{0} \in P_{\tilde{h}, \sigma}$, constructing successively the sequences

$$
\omega_{n}=\int_{0}^{1} G(t, s) \psi\left(s, \omega_{n-1}\right) d s-\sigma(t), \quad n=1,2, \ldots
$$

we have $\omega_{n} \rightarrow u^{*}$ as $n \rightarrow \infty$.

\section{Corollary 4.1 Suppose that condition $(H)$ holds and}

$\left(H_{5}^{\prime}\right) \psi:[0,1] \times[0,+\infty) \rightarrow(0,+\infty)$ is increasing with respect to the second variable, $a(t), b(t):[0,1] \rightarrow R^{+}$are continuous;

$\left(H_{6}^{\prime}\right)$ for any $\gamma \in[0,1]$, there exists $\varphi(\gamma)>\gamma$ such that

$$
\psi(t, \gamma x) \geq \varphi(\gamma) \psi(t, x), \quad \forall t \in[0,1], x \in(0,+\infty) .
$$

Then the following integral boundary value problem

$$
\left\{\begin{array}{l}
{ }_{0}^{R} \mathbb{D}_{t}^{\alpha, \lambda} u(t)+\psi(t, u(t))=0, \\
u(0)={ }_{0}^{R} \mathbb{D}_{t}^{\gamma_{1}, \lambda} u(0)={ }_{0}^{R} \mathbb{D}_{t}^{\gamma_{2}, \lambda} u(0)=\cdots={ }_{0}^{R} \mathbb{D}_{t}^{\gamma_{n-2}, \lambda} u(0)=0, \\
{ }_{0}^{R} \mathbb{D}_{t}^{\beta_{1}, \lambda} u(1)=\int_{0}^{\eta} b(s)_{0}^{R} \mathbb{D}_{t}^{\beta_{2}, \lambda} u(s) d A(s)+\int_{0}^{1} a(s){ }_{0}^{R} \mathbb{D}_{t}^{\beta_{3}, \lambda} u(s) d A(s)
\end{array}\right.
$$


has a unique positive solution $u^{*}$ in $P_{h}$ with $h=e^{-\lambda t} t^{\alpha-1}$. Moreover, for any given $\omega_{0} \in P_{h}$, constructing successively the sequences

$$
\omega_{n}=\int_{0}^{1} G(t, s) \psi\left(s, \omega_{n-1}(s)\right) d s,
$$

we have $\omega_{n}(t) \rightarrow u^{*}(t)$ as $n \rightarrow \infty$.

Proof Letting $c=0$ and $\rho=1$, from Theorem 4.1, we arrive at the conclusions.

\section{Applications}

To test our results established in the previous section, we provide two adequate problems.

Example 5.1 We investigate the tempered fractional differential system as follows:

$$
\left\{\begin{array}{l}
{ }_{0}^{R} \mathbb{D}_{t}^{\frac{5}{2}, \lambda} u(t)+f(t, u(t), u(t))+g(t, u(t))=0, \quad t \in(0,1) \\
u(0)={ }_{0}^{R} \mathbb{D}_{t}^{\frac{3}{4}, \lambda} u(0)=0, \\
{ }_{0}^{R} \mathbb{D}_{t}^{1, \lambda} u(1)=\int_{0}^{\eta}{ }_{0} \mathbb{D}_{t}^{\frac{5}{8}, \lambda} u(s) d A(s)+\int_{0}^{1}{ }_{0}^{R} \mathbb{D}_{t}^{\frac{7}{8}, \lambda} u(s) d A(s),
\end{array}\right.
$$

where $f(t, u, v)=(1-t)^{-\frac{1}{3}} t^{-\frac{2}{3}} u^{\frac{1}{3}}+v^{-\frac{1}{5}}, g(t, u)=(1-t)^{-\frac{1}{8}} t^{-\frac{1}{6}} u^{\frac{1}{3}}, \lambda=1>0, \eta=1$, and $A(t)=\frac{t}{2}$. For any $t \in(0,1), u>0$ and $v>0$. In system (5.1), we see that $\alpha=\frac{5}{2}, \beta_{1}=1, \beta_{2}=\frac{5}{8}, \beta_{3}=\frac{7}{8}$, $\gamma_{1}=\frac{3}{4}, a(t)=b(t) \equiv 1$.

Let us check that all the conditions of Theorem 3.1 are satisfied.

(1) Since $\frac{\Gamma\left(\alpha-\beta_{1}\right) e^{\lambda}}{\Gamma\left(\alpha-\beta_{2}\right)} \delta_{1}+\frac{\Gamma\left(\alpha-\beta_{1}\right) e^{\lambda}}{\Gamma\left(\alpha-\beta_{3}\right)} \delta_{2}=0.8221<1$, clearly, condition $(H)$ holds, and $f(t, u, v):(0,1) \times R^{+} \times R^{+} \rightarrow R^{+}$and $g(t, u):(0,1) \times R^{+} \rightarrow R^{+}$are continuous.

(2) It is evident that $f(t, u, v)$ is increasing in $u$ for fixed $t \in(0,1)$ and $v \in R^{+}$, decreasing in $v$ for fixed $t \in(0,1)$ and $u \in R^{+}$; in addition, for fixed $t \in(0,1), g(t, u)$ is increasing in $u$.

(3) For any $\gamma \in(0,1), t \in(0,1), u, v>0$, taking $\xi=\frac{1}{2}$, then $\gamma^{\xi} \in(\gamma, 1)$ and we obtain

$$
\begin{aligned}
f\left(t, \gamma u, \gamma^{-1} v\right) & =(1-t)^{-\frac{1}{3}} t^{-\frac{2}{3}}(\gamma u)^{\frac{1}{3}}+\left(\gamma^{-1} v\right)^{-\frac{1}{5}} \\
& =\gamma^{\frac{1}{3}}(1-t)^{-\frac{1}{3}} t^{-\frac{2}{3}} u^{\frac{1}{3}}+\gamma^{\frac{1}{5}} v^{-\frac{1}{5}} \\
& \geq \gamma^{\frac{1}{2}}\left[(1-t)^{-\frac{1}{3}} t^{-\frac{2}{3}} u^{\frac{1}{3}}+v^{-\frac{1}{5}}\right] \\
& =\gamma^{\xi} f(t, u, v)
\end{aligned}
$$

and

$$
\begin{aligned}
g(t, \gamma u) & =(1-t)^{-\frac{1}{8}} t^{-\frac{1}{6}}(\gamma u)^{\frac{1}{3}} \\
& =\gamma^{\frac{1}{3}}(1-t)^{-\frac{1}{8}} t^{-\frac{1}{6}} u^{\frac{1}{3}} \\
& \geq \gamma\left[(1-t)^{-\frac{1}{8}} t^{-\frac{1}{6}} u^{\frac{1}{3}}\right] \\
& =\gamma g(t, u) .
\end{aligned}
$$


(4) Taking $\delta_{0}=\frac{1}{2}$, for $\forall t \in(0,1)$ and $u, v \in[0,+\infty)$, we have

$$
\begin{aligned}
f(t, u, v) & =(1-t)^{-\frac{1}{3}} t^{-\frac{2}{3}} u^{\frac{1}{3}}+v^{-\frac{1}{5}} \\
& \geq \frac{1}{2}\left[(1-t)^{-\frac{1}{8}} t^{-\frac{1}{6}} u^{\frac{1}{3}}\right] \\
& =\delta_{0} g(t, u) .
\end{aligned}
$$

Now, all the assumptions of Theorem 3.1 are satisfied. Hence, Theorem 3.1 implies that $\operatorname{BVP}(5.1)$ has a unique positive solution $u^{*} \in P_{h}$, where $h(t)=e^{-t} t^{\alpha-1}$. Moreover, for any initial values $x_{0}, y_{0} \in P_{h}$, making successively the sequences

$$
\begin{aligned}
& x_{n}=\int_{0}^{1} G(t, s)\left((1-t)^{-\frac{1}{3}} t^{-\frac{2}{3}} x_{n-1}^{\frac{1}{3}}+y_{n-1}^{-\frac{1}{5}}\right) d s+\int_{0}^{1} G(t, s)(1-t)^{-\frac{1}{8}} t^{-\frac{1}{6}} x_{n-1}^{\frac{1}{3}} d s, \\
& y_{n}=\int_{0}^{1} G(t, s)\left((1-t)^{-\frac{1}{3}} t^{-\frac{2}{3}} y_{n-1}^{\frac{1}{3}}+x_{n-1}^{-\frac{1}{5}}\right) d s+\int_{0}^{1} G(t, s)(1-t)^{-\frac{1}{8}} t^{-\frac{1}{6}} y_{n-1}^{\frac{1}{3}} d s,
\end{aligned}
$$

we obtain $x_{n} \rightarrow u^{*}$ and $y_{n} \rightarrow u^{*}$ as $n \rightarrow \infty$.

Example 5.2 We consider the following integral boundary value problem:

$$
\left\{\begin{array}{l}
{ }_{0}^{R} \mathbb{D}_{t}^{\frac{9}{4}, \lambda} u(t)+\psi(t, u(t))=1, \quad t \in(0,1) \\
u(0)={ }_{0}^{R} \mathbb{D}_{t}^{\frac{1}{2}, \lambda} u(0)=0 \\
{ }_{0}^{R} \mathbb{D}_{t}^{\frac{3}{4}, \lambda} u(1)=\int_{0}^{\eta}{ }_{0}^{R} \mathbb{D}_{t}^{\frac{3}{8}, \lambda} u(s) d A(s)+\int_{0}^{1}{ }_{0}^{R} \mathbb{D}_{t}^{\frac{5}{8}, \lambda} u(s) d A(s)
\end{array}\right.
$$

where $\lambda=0, \eta=1, A(t)=\frac{t}{2}, \alpha=\frac{9}{4}, \beta_{1}=\frac{3}{4}, \beta_{2}=\frac{3}{8}, \beta_{3}=\frac{5}{8}, \gamma_{1}=\frac{1}{2}, a(t)=b(t) \equiv 1$. Evidently, $\frac{\Gamma\left(\alpha-\beta_{1}\right) e^{\lambda}}{\Gamma\left(\alpha-\beta_{2}\right)} \delta_{1}+\frac{\Gamma\left(\alpha-\beta_{1}\right) e^{\lambda}}{\Gamma\left(\alpha-\beta_{3}\right)} \delta_{2}=0.552<1$, condition $(H)$ is satisfied. In addition,

$$
\psi(t, u)=\left[\left(-\frac{8}{135 \Gamma\left(\frac{5}{4}\right)} u-\frac{2}{45 \Gamma\left(\frac{5}{4}\right)}\right) t^{\frac{9}{4}}+\left(m u+\frac{m}{8}\right) t^{\frac{5}{4}}\right]^{\frac{1}{3}}
$$

in which

$$
\begin{aligned}
& m=\frac{8}{15 \Gamma\left(\frac{5}{4}\right)}+\frac{352 \Delta^{-1}}{5175 \Gamma\left(\frac{5}{4}\right) \Gamma\left(\frac{15}{8}\right)}+\frac{32 \Delta^{-1}}{455 \Gamma\left(\frac{5}{4}\right) \Gamma\left(\frac{13}{8}\right)}, \\
& \Delta=\frac{1}{\Gamma\left(\frac{3}{2}\right)}-\frac{4}{2 \Gamma\left(15 \frac{15}{8}\right)}-\frac{4}{13 \Gamma\left(\frac{13}{8}\right)}>0
\end{aligned}
$$

Furthermore, we can get

$$
\begin{aligned}
\sigma(t)= & \int_{0}^{1} G(t, s) d s=\int_{0}^{1} G_{1}(t, s) d s+\frac{t^{\frac{5}{4}}}{\Delta \Gamma\left(\frac{15}{8}\right)} \int_{0}^{1} d s \int_{0}^{1} G_{2}(t, s) d \frac{t}{2} \\
& +\frac{t^{\frac{5}{4}}}{\Delta \Gamma\left(\frac{13}{8}\right)} \int_{0}^{1} d s \int_{0}^{1} G_{3}(t, s) d \frac{t}{2}
\end{aligned}
$$




$$
\begin{aligned}
& =\left(\frac{8}{15 \Gamma\left(\frac{5}{4}\right)}+\frac{352 \Delta^{-1}}{5175 \Gamma\left(\frac{5}{4}\right) \Gamma\left(\frac{15}{8}\right)}+\frac{32 \Delta^{-1}}{455 \Gamma\left(\frac{5}{4}\right) \Gamma\left(\frac{13}{8}\right)}\right) t^{\frac{5}{4}}-\frac{16}{45 \Gamma\left(\frac{5}{4}\right)} t^{\frac{9}{4}} \\
& =m t^{\frac{5}{4}}-\frac{16}{45 \Gamma\left(\frac{5}{4}\right)} t^{\frac{9}{4}}
\end{aligned}
$$

Setting $\tilde{h}(t)=\rho t^{\frac{5}{4}}$ with $\rho \geq 6$ and $t \in[0,1]$, it is easy to know $0 \leq \sigma(t) \leq 6 t^{\frac{5}{4}} \leq \rho t^{\frac{5}{4}}=\tilde{h}(t)$, $\sigma^{*} \leq 6$, and $\psi:[0,1] \times\left[-\sigma^{*},+\infty\right) \rightarrow R$ is continuous and increasing with respect to the second variable. In addition, let $\varphi(\gamma)=\gamma^{\frac{1}{3}}$ for $\gamma \in(0,1)$, we have

$$
\psi(t, \gamma u+(\gamma-1) v) \geq \varphi(\gamma) \psi(t, u), \quad \forall t \in[0,1]
$$

where $u \in(-\infty,+\infty), v \in\left[0, \sigma^{*}\right]$. Now, all the assumptions of Theorem 4.1 are satisfied, therefore, we can have a clear mind that BVP (5.2) has a unique nontrivial solution in $P_{\tilde{h}, \sigma}$, and for $\forall u_{0} \in P_{\tilde{h}, \sigma}$, let

$$
\begin{aligned}
u_{n}= & \int_{0}^{1} G(t, s)\left(\left(-\frac{8}{135 \Gamma\left(\frac{5}{4}\right)} u_{n-1}(s)-\frac{2}{45 \Gamma\left(\frac{5}{4}\right)}\right) s^{\frac{9}{4}}+\left(m u+\frac{m}{8}\right) s^{\frac{5}{4}}\right)^{\frac{1}{3}} d s \\
& +\frac{16}{45 \Gamma\left(\frac{5}{4}\right)} t^{\frac{9}{4}}-m t^{\frac{5}{4}}
\end{aligned}
$$

we have $u_{n} \rightarrow u^{*}$ as $n \rightarrow \infty$.

\section{Conclusion}

A fractional derivative (or integral) is a convolution with a power law, and a tempered fractional derivative multiplies that power law kernel by an exponential factor. Furthermore, the tempered fractional differential equations models open up a new kind of possibility for robust mathematical modeling of complex multi-scale problems and anomalous phenomena. Therefore, the emerging theory of tempered fractional calculus provides a sound mathematical basis for applications.

\section{Acknowledgements}

We are thankful to the editor and anonymous reviewers for many valuable suggestions to improve this paper

\section{Funding}

This paper is supported by the opening project of State Key Laboratory of Explosion Science and Technology (Beijing Institute of Technology). The opening project number is KFJJ19-06M. And it is also supported by the Key R\&D Program of Shanxi Province (International Cooperation, 201903D421042)

Availability of data and materials

Data sharing not applicable to this article as no datasets were generated or analysed during the current study.

Competing interests

The authors declare that they have no competing interests.

Authors' contributions

All authors contributed equally to the manuscript and typed, read, and approved the final manuscript.

\section{Author details}

'College of Mathematics, Taiyuan University of Technology, Taiyuan, P.R. China. ${ }^{2}$ Department of Mathematics, Lvliang University, Lvliang, P.R. China. ${ }^{3}$ State Key Laboratory of Explosion Science and Technology, Beijing Institute of Technology, Beijing, P.R. China. 


\section{Publisher's Note}

Springer Nature remains neutral with regard to jurisdictional claims in published maps and institutional affiliations.

\section{Received: 7 February 2020 Accepted: 28 April 2020 Published online: 12 May 2020}

\section{References}

1. Zhang, H.: Iterative solutions for fractional nonlocal boundary value problems involving integral conditions. Bound. Value Probl. 2016, 3 (2016)

2. Cheng, C., Feng, Z., Su, Y.: Positive solutions for boundary value problem of fractional differential equation with derivative terms. Electron. J. Qual. Theory Differ. Equ. 2012, 215 (2012)

3. Weitzner, H., Zaslavsky, G.M.: Some applications of fractional equations. Commun. Nonlinear Sci. Numer. Simul. $8(3-4), 273-281$ (2003)

4. Zhou, B., Zhang, L.: Existence of positive solutions of boundary value problems for high-order nonlinear conformable differential equations with $p$-Laplacian operator. Adv. Differ. Equ. 2019, 351 (2019)

5. Caputo, M., Fabrizio, M.: A new definition of fractional derivative without singular kernel. Prog. Fract. Differ. Appl. 1(2), 73-85 (2015)

6. Aydogan, S.M., Baleanu, D., Mousalou, A., Rezapour, S.: On approximate solutions for two higher-order Caputo-Fabrizio fractional integro-differential equations. Adv. Differ. Equ. 2017, 221 (2017)

7. Baleanu, D., Mousalou, A., Rezapour, S.: The extended fractional Caputo-Fabrizio derivative of order $0 \leq \sigma<1$ on $C_{\mathbb{R}}[0,1]$ and the existence of solutions for two higher-order series-type differential equations. Adv. Differ. Equ. 2018, $255(2018)$

8. Baleanu, D., Jajarmi, A., Mohammadi, H., Rezapour, S.: A new study on the mathematical modelling of human liver with Caputo-Fabrizio fractional derivative. Chaos Solitons Fractals 134, 109705 (2020)

9. Baleanu, D., Mousalou, A., Rezapour, S.: On the existence of solutions for some infinite coefficient-symmetric Caputo-Fabrizio fractional integro-differential equations. Bound. Value Probl. 2017, 145 (2017)

10. Baleanu, D., Ghafarnezhad, K., Rezapour, S., Shabibi, M.: On the existence of solutions of a three step crisis integro-differential equation. Adv. Diff. Eq. 2018, 135 (2018)

11. Goufo, E.F.D., Kumar, S., Mugisha, S.B.: Similarities in a fifth-order evolution equation with and with no singular kernel. Chaos Solitons Fractals 130, 109467 (2020)

12. Aydogan, S.M., Baleanu, D., Mousalou, A., Rezapour, S.: On high order fractional integro-differential equations including the Caputo-Fabrizio derivative. Bound. Value Probl. 2018, 90 (2018)

13. Baleanu, D., Rezapour, S., Saberpour, Z:: On fractional integro-differential inclusions via the extended fractional Caputo-Fabrizio derivation. Bound. Value Probl. 2019, 79 (2019)

14. Baleanu, D., Rezapour, S., Mohammadi, H.: Some existence results on nonlinear fractional differential equations. Philos. Trans. R. Soc. 371, 20120144 (2013)

15. Baleanu, D., Mohammadi, H., Rezapour, S.: Analysis of the model of HIV-1 infection of CD4+ T-cell with a new approach of fractional derivative. Adv. Differ. Equ. 2020, 71 (2020)

16. Alizadeh, S., Baleanu, D., Rezapour, S.: Analyzing transient response of the parallel RCL circuit by using the Caputo-Fabrizio fractional derivative. Adv. Differ. Equ. 2020, 55 (2020)

17. Baleanu, D., Ghafarnezhad, K., Rezapour, S.: On a three step crisis integro-differential equation. Adv. Differ. Equ. 2019 153 (2019)

18. Zaky, M.A.: Existence, uniqueness and numerical analysis of solutions of tempered fractional boundary value problems. Appl. Numer. Math. 3537, 1-29 (2019)

19. Zhai, C., Hao, M.: Fixed point theorems for mixed monotone operators with perturbation and applications to fractional differential equation boundary value problems. Nonlinear Anal. 75, 2542-2551 (2012)

20. Zhang, X., Zhong, Q.: Uniqueness of solution for high-order fractional differential equations with conjugate type integral conditions. Fract. Calc. Appl. Anal. 20(6), 1471-1484 (2012)

21. Zhang, X., Liu, L., Wu, Y.: The eigenvalue problem for a singular fractional differential equation involving fractional derivative. Appl. Math. Comput. 218, 8526-8536 (2013)

22. Min, D., Liu, L., Wu, Y.: Uniqueness of positive solutions for the singular fractional differential equations involving integral boundary value conditions. Bound. Value Probl. 2018, 23 (2018)

23. Zhai, C., Wang, L.: $\varphi-(h, e)$-Concave operators and applications. J. Math. Anal. Appl. 454, 571-584 (2017)

24. Zhai, C., Hao, M.: Mixed monotone operator methods for the existence and uniqueness of positive solutions to Riemann-Liouville fractional differential equation boundary value problems. Bound. Value Probl. 2013, 85 (2013)

25. Zhou, B., Zhang, L.: Multiple positive solutions for nonlinear high-order Riemann-Liouville fractional differential equations boundary value problems with $p$-Laplacian operator. Bound. Value Probl. 2020, 26 (2020). https://doi.org/10.1186/s13661-020-01336-1 